INTERNATIONAL DESIGN CONFERENCE - DESIGN 2018

https://doi.org/10.21278/idc.2018.0491

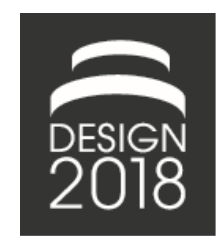

\title{
NEW EYES OF ID - HOW TO PREPARE NEW INDUSTRIAL REVOLUTION AS INDUSTRIAL DESIGNER
}

\author{
E.-C. Jung, J. M. Choi and Y. Sim
}

\begin{abstract}
At the turning point of technological innovation, an intriguing question is how industrial designers can bridge gap between human and technology. The role of the industrial designer in the traditional view of the physical appearance of objects may be reduced, but in the future, designing meaning with object through rich interaction is becoming a wiser way of preparing for a new industrial revolution. The purpose of this paper is to discuss the changing role of designers through theoretical reviews with case studies.
\end{abstract}

Keywords: industrial design, human centred design, user experience, interaction design

\section{Motivation and goal}

Mullainathan (2009) called the failure of newly developed technology integration the "last mile problem" in a 2009 TED Talk. Now, at the turning point of technological innovation (Toffler, 1980), IT and energy (Rifkin, 2011), IoT (Internet of Things) (Ashton, 2009) and intellectual labor (Brynjofsson and McAfee, 2014), an intriguing question is how industrial designers can solve the last mile problem. Technology innovation does not lead directly to solutions. The fact that design considers meanings to address inconsistency between human and technology, with the external form, has been discussed from a variety of perspectives. Sparke (1986) has linked the history of design to changes in production technology and systems, and noted the inconsistency of the dual relationship between mass production and consumption. Baudrillard (1968) described the need to understand objects in the way they are consumed, and explained the system of things as an analysis of the system of semantics in which human beings and things are related. Form and meaning are complex, but their relationship is a fundamental concern of the product semantic. Things must have a visible form, but they must be valid for being understood and used (Krippendorff, 1989).

The role of design can be explained by the fact that technology innovation promotes the process of forming the meaning through human interaction. Frens (2006) argues that design changes into handcrafted, mechanical, electrical, and electronic products according to the innovation of technology. These changes shows that meaning of the object by industrial design transforms to form, control and coupling. In hand-crafted products, form was the most important mediator of meaning. However, in electronic products, coupling between technology and controls is critical for users to understand function and meaning of the products. Dourish (2004) described coupling as a concept connecting perceptions to execution, and they explained that the embodied skills depend on a tight coupling between perception and action. Loosely, proximal means close by or at hand, while distal means at a distance. Therefore, the role of the industrial designer in the traditional view of the physical appearance of the object may be reduced, but in the future, it is a correct interpretation that designing meaning 
with object through rich interaction is becoming more wise eyes to prepare new industrial revolution. At the present time, which is being talked about in the era of the new industrial revolution, the purpose of this paper is to discuss the role of the changing designer through the case study of the designed objects and the perspective of the industrial designer to view the design in order to narrow the inconsistency of human and technology.

\section{Framework: Toward form, emotion to meaning}

In order to achieve the goal of this research, a framework in (Figure 1) based on the relationship between human, technology and design is suggested to describe how design play a role between human and technology. 1) In the case of humans, Abraham Maslow (Maslow, 1943) stated that human desires consist of five stages: physiological safety love esteem self actualization. And, when the subordinate desires are satisfied, they have a tendency to gradually go to the upper desires. 2) In the case of technology, based on Martin Heidegger's view of technology, Carl Mitcham (Mitcham, 1994) discussed types of technology as Object-Knowledge-Activity-Volition, and David M. Berry (Berry, 2011) added the stage of phenomenon on the top layer of technology that Mitcham asserted, and argued for the change of technology and social phenomenon gradually developed to the upper level based on the technologies belonging to the subordinates.

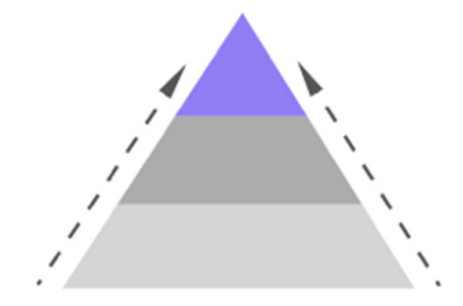

Maslow's hierarchy of needs
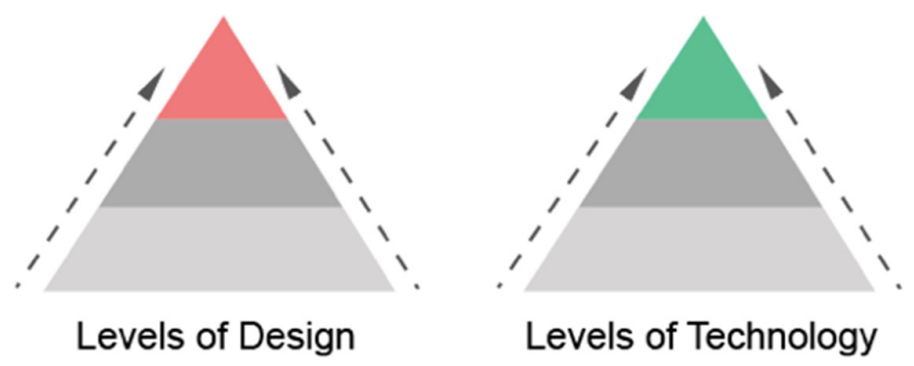

TECHNOLOGY

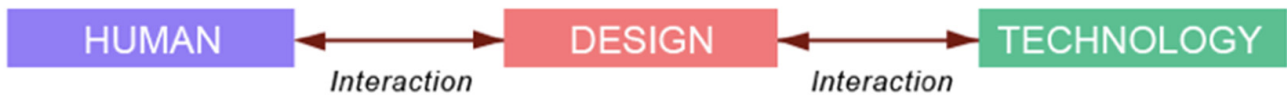

Figure 1. Framework based on the relationship between human, design and technology

3) Design is a medium that helps human beings and technology to dissolve into life in society, and also it interacts with the developmental stage of technology and the psychological stage of human beings. According to Steven Bradley (Bradley, 2010), the design is described in the level of Functionality-Reliability-Usability-Proficiency-Creativity. In the study of Lucia Rampino (Rampino, 2011), the design is classified as anAesthetic and Usability-Meaning-Typology. Aarron Walter (Walter, 2011) advocated changes in subordinate concept and superordinate concepts according to the development of human and technology as a stage of design functional-reliableusage-pleasurable experience. With the emergence of transcendental technology claimed by future social and technological industries will progress, human behavior will change together, and the role that design has to solve gradually has to go to the upper level. Therefore, the designers should continue to challenge and research new directions.

Industrial designers must cope with a new perspective on the upcoming future and a broader worldview in order to be ready for the case the future environment encroaches upon past and present environments and products. Therefore, by looking at the stages of the psychological essence of humans and the development stages of technology, it is possible to suggest a new interpretation of the design stage. It can also be an opportunity to see why the direction of design should be directed to the top will be. The framework of (Figure 1) could provide holistic perspectives for finding answers as industrial designers. 


\section{Perspectives}

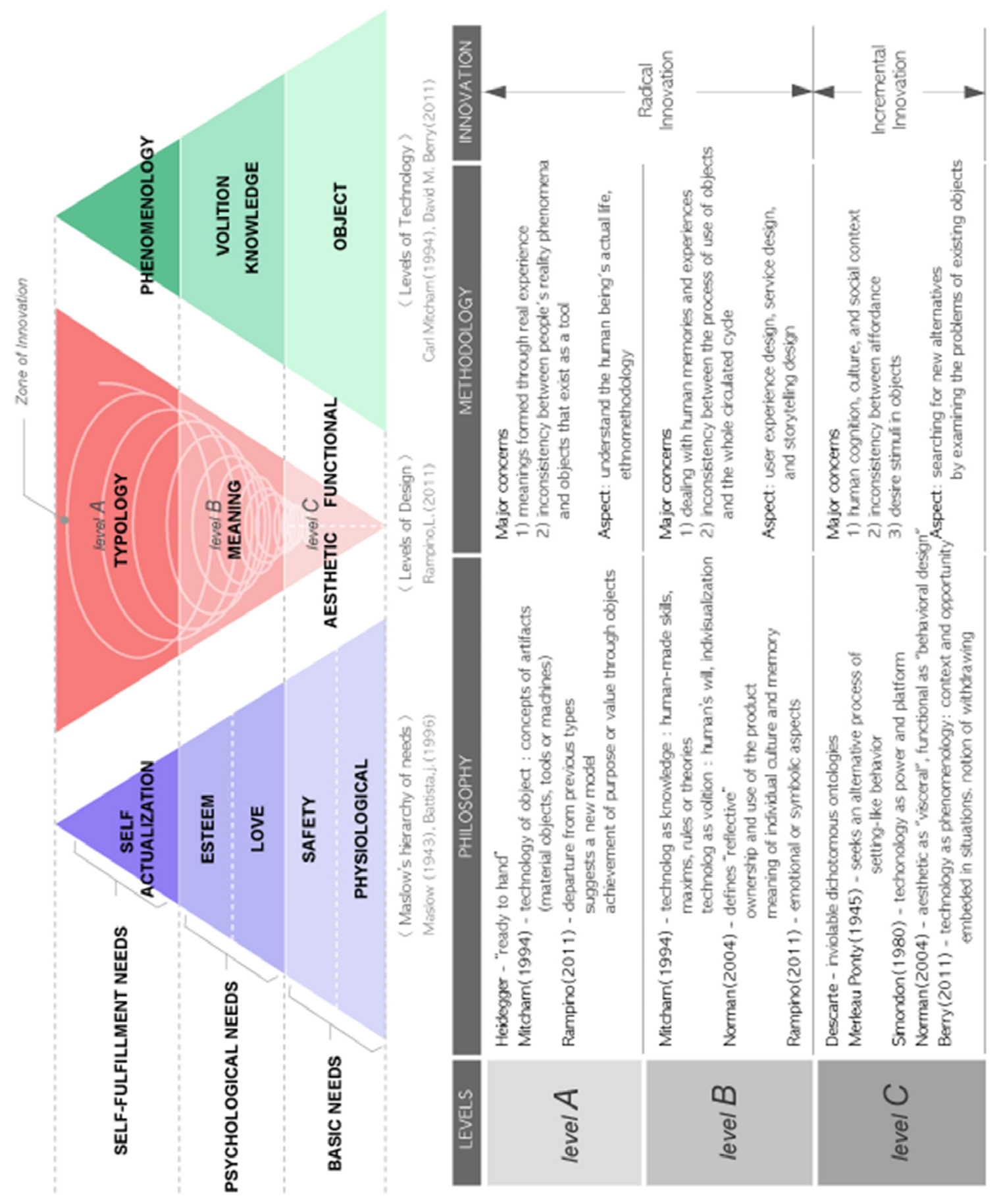

Figure 2. The design phase is set in levels $a, b$, and c along with the levels of the technology, and the philosophy, methodology, and innovation type are described in detail for explaining "Levels of design"

Efforts to narrow the inconsistency between technology applied to objects and humans have been studied extensively in the field of design and technology. In order to examine the achievements of this 
study more specifically, the contents of (Figure 2) are composed by adding various aspects, such as specific contents, philosophical perspectives, and innovation type, to (Figure 1) which describes the design and technology pyramid model. The model of (Figure 2) assumed the development of technology in connection with human needs, and design is served as a bridge between them. To illustrate that the level A produces bigger impacts, the inverted triangle is used for design. In (Figure 2), we examine the technology applied to objects designed by projecting human needs and the technology applied to objects that create new socio-cultural phenomena. We will also discuss the perspectives that will allow us to think about the future vision and role of product designers.

\section{Level C}

This step is described in terms of aesthetic and functional improvement of the product in the design (Rampino, 2011). Material objects, i.e., tools and machines, which are concepts of artifacts created by humans through technology, are described in terms of technology as objects (Mitcham, 1994). This stage is based on the notion that Descartes' human and objects are inviolable dichotomous ontologies. According to Merleau-Ponty, a process by which the human body escapes from habitual behavior and seeks an alternative process of setting-like behavior (Merleau-Ponty, 1945). Norman (2004) defines aesthetic as "visceral" and functional as "behavioral design". The methodological perspective of this level seeks new alternatives by examining the problems of existing objects from various perspectives. Considering human cognition, culture, and social context, and the inconsistency between affordance and desire stimuli in objects, is a major concern of design, and this level may be explained by incremental innovation (Dell'Era and Verganti, 2007). Product designers are familiar with these perspectives for product designers, and many things used in daily life are being designed from this perspective.

\section{Level B}

This stage can be described in terms of the meaning of things through emotional or symbolic aspects of design (Rampino, 2011), and in terms of efficiency, process of forming meaning, and knowledge and volition in the field of technology. Technology as knowledge can be proven by the skills, maxims, rules or theories created by man, taking into account making and using of artifacts. The technology as volition is to return to philosophy from engineering. Due to the inclusion of 'willing' in humans, this is a characteristic that is difficult to find in earlier stages of technology. Thus, this is the most individualized and subjective of the modes of technology, and this personality rarely shows popular consequences (Mitcham, 1994). This is based on the semantics of emotions and symbols, and Norman (2004) describes this level as reflective. The product of this stage forms the meaning of the existence of the thing through overall quality and story delivery by communication (Rampino, 2011). In other words, the experience of ownership and use of the product are emphasized, and the meaning of individual culture and memory becomes important (Norman, 2004). The main concern of design is dealing with human memories and experiences that actually exist, and the inconsistency between the process of use of objects and the whole circulated cycle. Our everyday objects and the process of reflection of these objects are being designed in terms of user experience design, service design, and storytelling design.

\section{Level A}

Design and technology have pursued each other as power, but they are reaching the same point of view. For example, the design of Salone del Mobile and the Consumer Technology Association (CES) are exhibitions that represent design and technology, both of which have a similar ultimate goal. Therefore, in the future, we can expect to hold an exhibition in which technology and design are united. Human beings and technology are naturally assimilated and embodied. Heidegger explains it as "ready to hand" explaining it as invisibility, that is, the concept of disappearing things as a tool that enable us to focus on the ultimate goal. Berry (2011) expressed this as a phenomenology, claiming that the phenomenon is left because it focuses on the experience of 'forgetting' technology. Technology as phenomenology constantly reminds us of the importance of reference to the combined meaning of social context and things, and this refers to looking at the ways and opportunities involved in the situation. In order to do this, it is important to understand the human being's actual life, and a methodological approach that can be explained by the human experience pursued by ethnomethodology (Garfinkel, 1967). Typology Innovation, based on this, is a departure from previous types of products; which suggests a new model, and the achievement of purpose or value through objects is more important than. The main concern of designers is to look at the meanings formed through real experience, and inconsistency between people's 
reality phenomena and objects that exist as tools. This rarely happens, but has bigger impact as seen in 'Zone of Innovation' in Figure 2.

\section{Case studies}

Based on the perspectives of (Figure 2), we used two case studies to explain why the role of industrial designer is becoming more and more critical and how the spiral evolution leads influential changes. Projector is becoming being pervasive as an important element of smart home based on IoT, and starting from the awareness of environment and energy, the electric vehicle is evolving into a radical innovation. For this reason, new technologies that are currently underway, examples to be introduced in the new era of industrial revolution and technology, are meaningful examples that can redefine the role of industrial designers.

\subsection{Projector}

A projector projects images onto a wall, and it was invented to view movies and enlarge images in homes and cinemas. Initial projectors "magic lantern" was invented by a German priest called Athanasius Kircher in 1646, which used candlelight as light source to make the religious inspiration even greater. After that, 'Filmstrip Projector' was released in the 1920s and was used for teaching in class time.

As an example of the Level C viewpoint, projectors developed from the viewpoint of objects such as tools and machines were commercialized and used for office work and education in the early days. Due to frequent theft, they applied a uniform color design to every public projector in (Figure 3), which is a product design with creative solutions to the problem of theft. In the Level B perspective, as the needs of the projector evolve from the public to the personalization, the user experience of watching movies and creating atmosphere at home gradually spreads, and it develops into a design in (Figure 4) that can enjoy video with friends and family user experience of watching the movie or creating the atmosphere, which can help you enjoy movies with friends and family at home or outdoors. In other words, inconsistency between the new experience created by the projector and the existing projector have created the emergence of new product designs. Level A is a Sony 4K Ultra Short Throw Projector in (Figure 5) introduced at CES in 2017 as an example that focuses on design and technology with the same innovative point. This projector is one of thean element of the smart home and is a case in which the projector presents a new typology. This projector is connected to other devices and a network, and its form is vanished. This creates a natural opportunity by being embedded in the situation, so that it plays a variety of roles such as TV and digital picture frames in the home, and it also connects with wind information of the air conditioner, so that a more realistic presentation becomes possible. When it is off, it offers more new possibilities to the space occupied by existing TV. In the smart home, various electronic devices connected to one network have broken down the barriers among devices. In this connected ecosystem, such as checking the condition of the refrigerator on the TV and turning on the air conditioner, the projector no longer has a single screen but also play a role as a center to control the ecosystem.

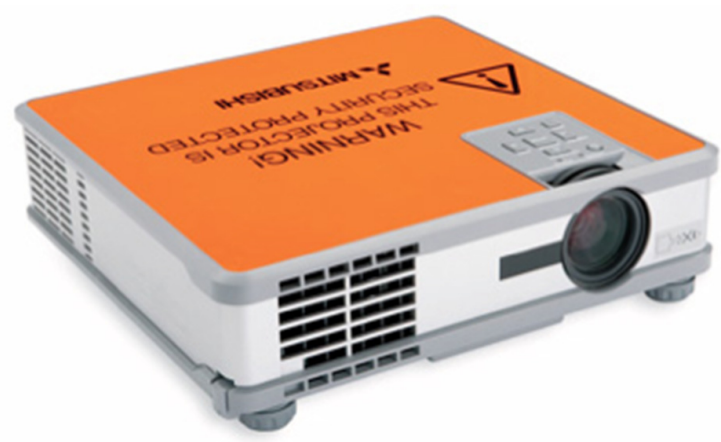

Figure 3. Mitsubishi XL6U-Orange Projector; As an example of level C (Retrieved from http://www.dreamav.co.uk/mitsubishi-xl6u-projector.htm) 


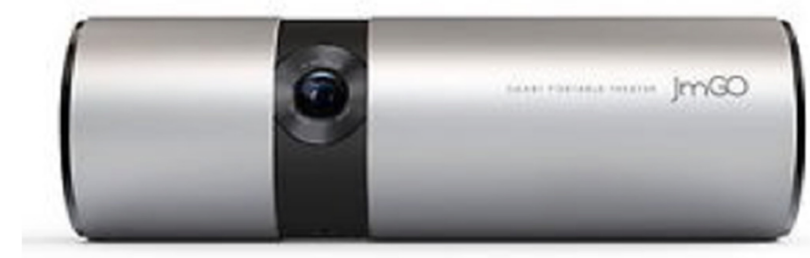

Figure 4. JmGO P2 View Portable Projector; As an example of level B (Retrieved from http://www.ebay.com/itm/JmGO-View-Smart-Portable-ProjectorSupport-1080P-Hi-Fi-Bluetooth-/152214289032)

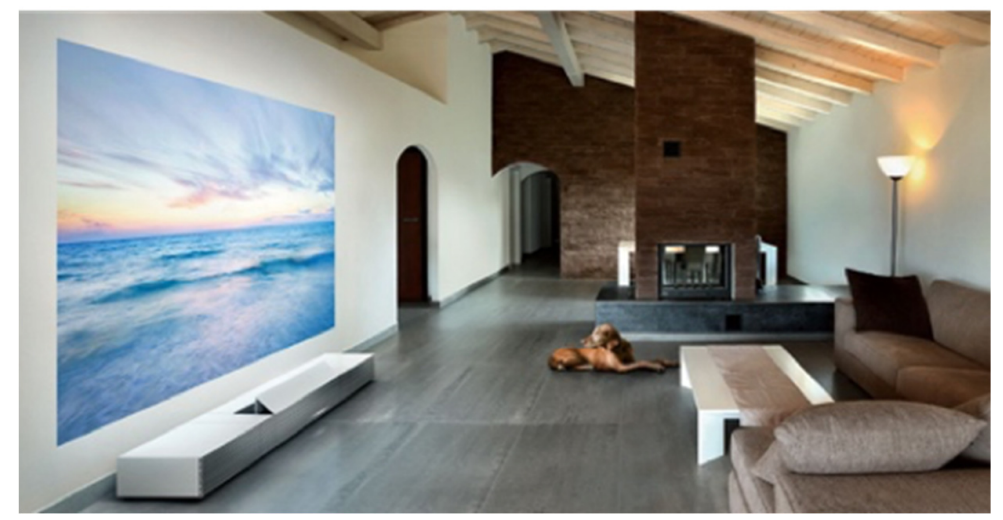

Figure 5. Sony 4K Ultra Short Throw Projector; As an example of level A (Retrieved from http://www.sony.com/electronics/projector/lspx-w1s)

\subsection{BMW MINI}

In 1956, the Sues Crisis caused gasoline prices skyrocketing in England. As gasoline prices rose higher than ever, there was a need to develop a car with low fuel costs. To meet the needs, BMC (British Motor Corporation) developed a car with compact size, low fuel cost, and space efficiency- the MINI. In Level $\mathrm{C}$ point of view, MINI has achieved a functional improvement in fuel efficiency and space efficiency, which is the basic function of a car. In order to make these functional improvements, MINI came up with an innovative layout: pushing the wheels all the way out to the corners and turning the engine sideways. This innovative layout extended space efficiency to the extreme, and it is still adopted by most of compact cars nowadays.

In 1960, the MINI, recreated by John Cooper, won several competitions as a race and rally car. This draw attention from the public, and more importantly, celebrities like Paul McCartney, George Harrison, Steve McQueen and Peter Sellers purchased MINI along with bigger and much expensive cars in (Figure 6). This led to step Level B, in that people chose MINI as means for self-expression, not as an economical transportation option. Peter Seller was one of those that led this trend with his customized MINI, and Mary Quant, the so-called mother of the miniskirt, designed a dress inspired by MINI and customized her car to match the style. However, what made MINI so popular in the 60's was the due to the hit of the British film, Italian Job (1967) in (Figure 8). In the film, mini was depicted as a small but powerful and nimble car, in the scene of the car chase rally against the police. MINI's depiction as a spirit of freedom adventure in the film was in line with the 60's rebel against parental value. During this time, MINI's slogan "You don't need a big one to be happy" caught minds of youngsters in (Figure 7), as it represented the soul of freedom and rebellion, which was the essence of the 60's youth culture. From then, MINI was not a mere car; it came to represent the icon of the era.

However, as time changes and the spirit of " 60 s fades away, MINI also saw decline in the sales. New competitors like Austin/MG Metro made MINI look outdated and MINI's production became unstable. Nevertheless, BMW bought the Rover Group, and regenerated MINI in 2000. This became a new turning point for MINI. With components manufactured by BMW, MINI was armed with the latest 
technologies like the powerful turbo engine, head-up display for drivers' convenience, and parking assistance, along with the luxurious exterior which gave MINI a new position as a premium compact car. However, what people are enthusiastic about MINI is its position as a cultural icon. In Level A point of view, MINI nowadays sells its identity, not the car itself as a means of transportation. After the debut of second generation MINI, MINI introduced various lineups- clubman, countryman, cooper, convertible and so on- but all of them shares the one MINI identity in in (Figure 9). Moreover, as a sociocultural icon, MINI appears in various kinds of items, which represents the MINI style. The famous example of this is a bag with MINI printed on it, which is produced by collaboration with the British fashion icon Paul Smith in (Figure 10). In other words, BMW recreated MINI by adding new technology, while preserving its original identity. This enabled MINI to break the presumption that small cars are cheap, and gave MINI a new meaning as a sociocultural phenomenon.

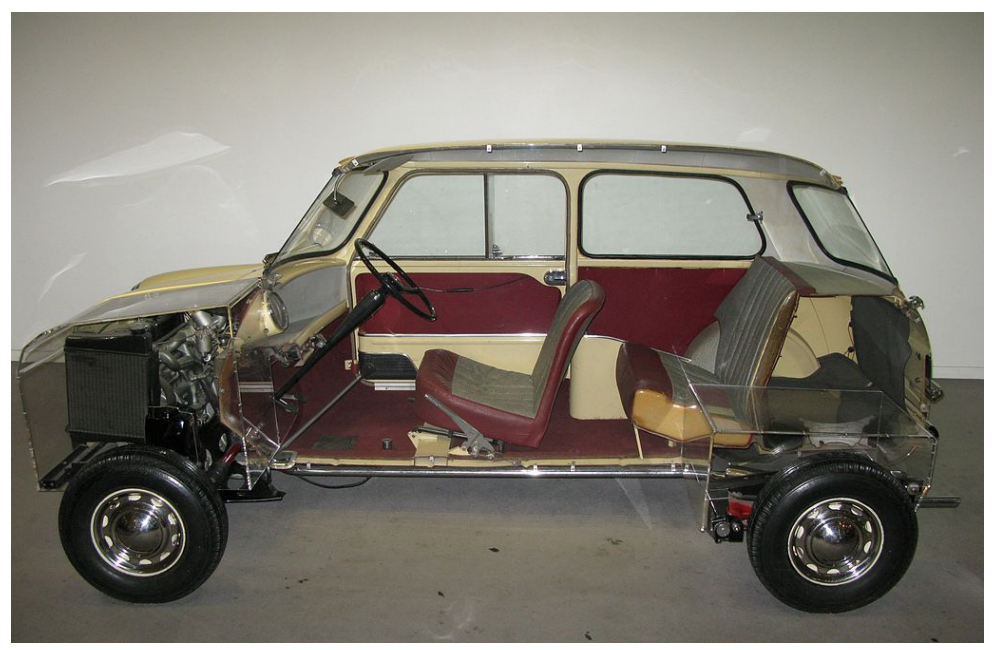

Figure 6. Cross section of MINI; As an example of level C (Retrieved from https://en.wikipedia.org/wiki/Economy_car)

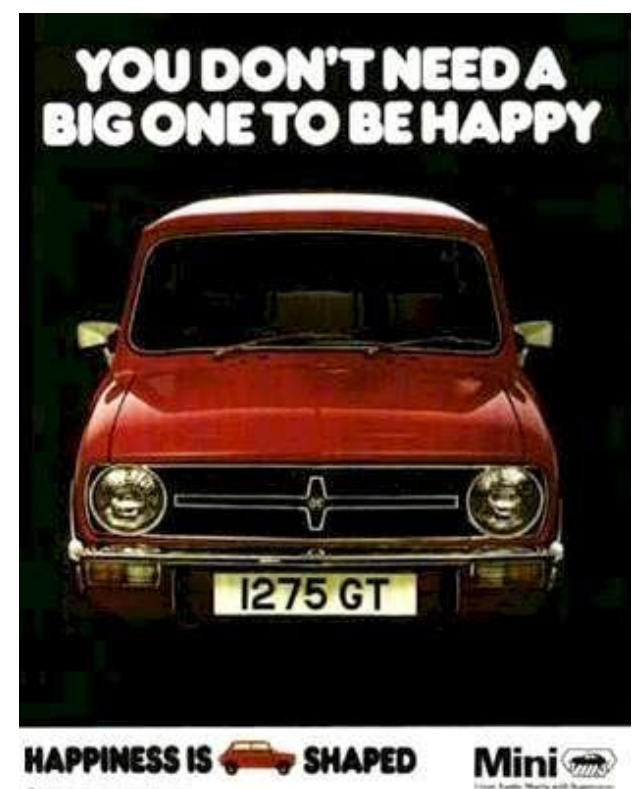

Figure 7. '60s MINI slogan; As an example of level B (Retrieved from https://www.flickr.com/photos/ohsoretroevents/13168190283) 


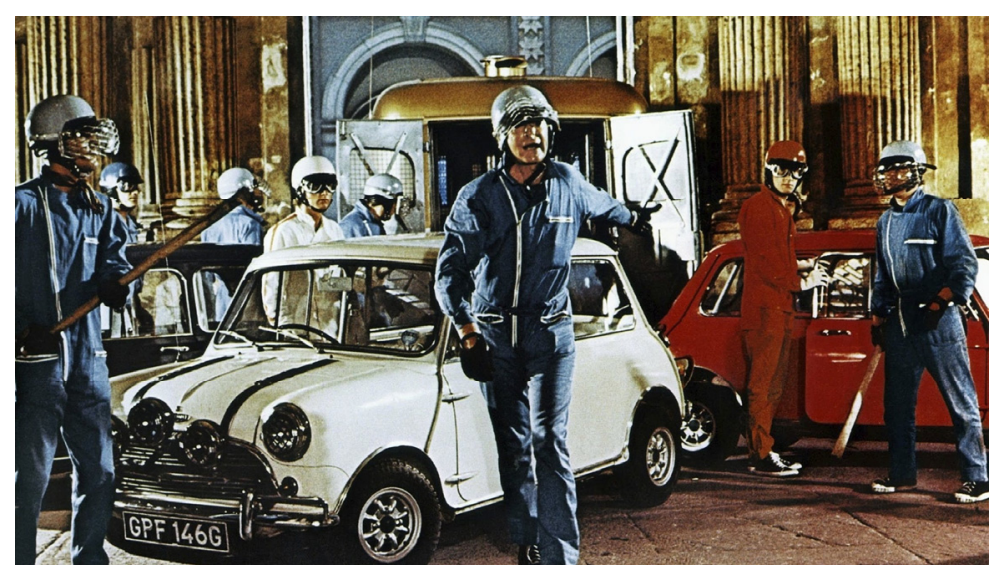

Figure 8. Italian Job (1967); As an example of level B

(Retrieved from https://drivetribe.com/p/the-italian-job-mini-cooper-s-

CYHYDoYWRE-FP1TAYuD1RQ?iid=Vj_oQYYWSue21ZDzkNHAJw)

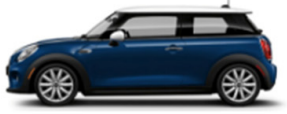

MINI HARDTOP 2 DOOR

Starting at $\$ 21,600^{*}$

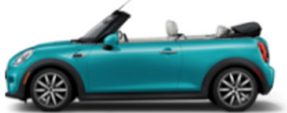

MINI CONVERTIBLE Starting at $\$ 26,700^{*}$

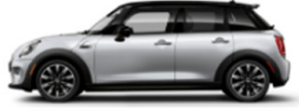

MINI HARDTOP 4 DOOR

Starting at $\$ 22,600^{*}$

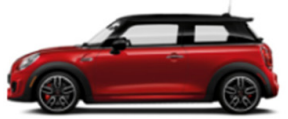

JOHN COOPER WORKS See the Full Line-Up

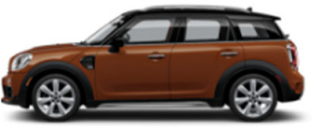

MINI COUNTRYMAN

Starting at $\$ 26,600^{*}$

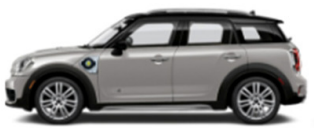

$\bigoplus$ MINI ELECTRIC

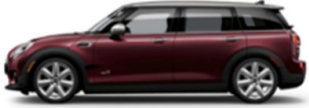

MINI CLUBMAN

Starting at $\$ 24,800^{*}$

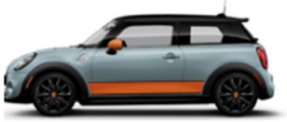

SPECIAL EDITIONS Ice Blue \& More

Figure 9. MINI lineups: As an example of level A (Retrieved from http://www.miniusa.com/content/miniusa/en.html)

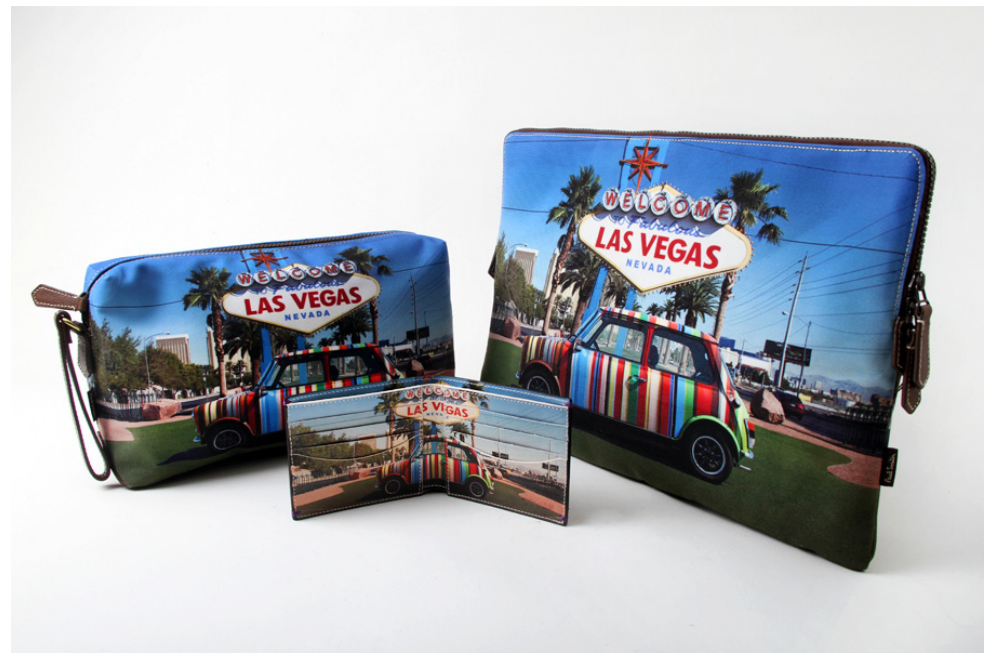

Figure 10. Collaboration item with Paul Smith; As an example of level A (Retrieved from http://www.luxuo.com/culture/design/paul-smith-mini-onlocation-accessories.html) 


\section{Discussion and conclusion}

The dematerialization of objects and media has created a crisis of consciousness about the role of industrial designers. In fact, the position of design is narrowing down to the role of designing such as frame and pedestal of television. Tangible and physical interaction design can be seen as an experiment to overcome this phenomenon. In this research, we have to reconsider the relationship between the development of technology and industrial design and discuss the role of industrial designers. As we have seen in these two case studies, industrial designers are expected to propose a product concept that does not exist based on accumulated memories, and create a typology of life. Suzanne LaBarre's (LaBarre, 2016) "The Most Important Design Jobs of the Future" predicts that designers will have to present a memory and experience of Type B and new lifestyle of Type A. Industrial designers are expected to have eyes to bridge humans and technology to embody the human experience in the virtual realm, objects, and space. For this change, industrial design education should not only focus on the form of things. Industrial designer must design behaviors by understanding the meaning of technology from a human perspective. The "last mile problem" mentioned by Mullainathan will be overcome with new eyes and are cautiously optimistic that industrial designers will play a pivotal role in the development of human culture.

\section{Acknowledgments}

This work was supported by the Ministry of Education of the Republic of Korea and the National Research Foundation of Korea (NRF-2016S1A5A2A01927912).

\section{References}

Ashton, K. (2009), “That 'Internet of Things' Thing”, RFID Journal, Vol. 22 No. 7, pp. 97-144.

Baudrillard, J. (1968), Le systome des objets, Gallimard, Paris.

Berry, D.M. (2011), The Philosophy of Software: Code and Mediation in the Digital Age, Palgrave Macmillan London. https://doi.org/10.1057/9780230306479

Bradley, S. (2010), Designing For A Hierarchy Of Needs. [online] Smashing magazine. Available at: https://www.smashingmagazine.com/2010/04/designing-for-a-hierarchy-of-needs/ (accessed 09.13.2017).

Brynjofsson, E. and McAfee, A. (2014), The Second Machine Age: Work, Progress, and Prosperity in a Time of Brilliant Technologies, W. W. Norton \& Company, New York.

Dell'Era, C. and Verganti, R. (2007), "Strategies of innovation and imitation of product languages", Journal of Product Innovation Management, Vol. 24 No. 6, pp. 580-599. https://doi.org/10.1111/j.15405885.2007.00273.x

Design council (2011), Designing out crime- a designer's guide. [online] Design council. Available at: http://www.designcouncil.org.uk/sites/default/files/asset/document/designersGuide_digital_0_0.pdf(accessed 09.13.2017).

Dourish, P. (2004), Where the Action Is; The Foundations of Embodied Interaction, The MIT Press, Cambridge.

Frens, J.W. (2006), Designing for rich interaction - Integrating form, interaction and function, PhD thesis, Eindhoven University of Technology.

Garfinkel, H. (1967), Studies in Ethnomethodology, Polity Press, Cambridge.

JmGO (2017), JmGO projector. [online] JmGO. Available at: http://en.jmgo.com/product/p2 (accessed 09.13.2017).

Krippendorff, K. (1989), "On the essential contexts of artifacts or on the proposition that design is making sense (of things)”, Design Issues, Vol. 5 No. 2, pp. 9-39. https://doi.org/10.2307/1511512

LaBarre, S. (2016), The Most Important Design Jobs of the Future. [online] Fast Company Magazine. Available at: https://www.fastcodesign.com/3054433/the-most-important-design-jobs-of-the-future (accessed 11.13.2017).

Maslow, A.H. (1943), “A theory of human motivation”, Psychological Review, Vol. 50 No. 4, pp. 370-396. https://doi.org/10.1037/h0054346

Merleau-Ponty, M. (1945), Phenomenologie de la perception, Gallimard, Paris.

MINI USA (2017), The (short) story of MINI. [online] MINI USA. Available at: http://www.miniusa.com/content/miniusa/en/why-mini/why-mini/over-50-years-of-motoring.html (accessed 11.05.2017).

Mitcham, C. (1994), Thinking through technology, The University of Chicago Press, Chicago.

Norman, D. (2004), Emotional design, Apogeo, Milano. 
Rampino, L. (2011), “The Innovation Pyramid: A Categorization of the Innovation Phenomenon in the Productdesign Field”, International Journal of Design, Vol. 5 No. 1, pp. 3-16.

Rifkin, J. (2011), The Third Industrial Revolution: How Lateral Power Is Transforming Energy, the Economy, and the World, St. Martin's Griffin, New York.

SONY (2017), LSPX-WIS $4 K$ Ultra Short Throw Projector. [online] SONY. Available at: http://www.sony.com/electronics/projector/lspx-w1s (accessed 09.13.2017).

Sparke, P. (1986), An Introduction to Design and Culture in the Twentieth Century, Routledge, London.

Toffler, A. (1980), The Third Wave, Morrow, New York.

Walter, A. (2011), Designing for Emotion, A Book Apart, New York.

Prof. Eui-Chul Jung, Associate Professor

Seoul National University, Department of Design

YeSulkwan \#49-303,1 Gwanak-ro, Gwanak-gu, 151-742 Seoul, South Korea

Email: jech@snu.ac.kr 